Financial and proprietary interest: Nil

Financial support: Nil

Eye (2006) 20, 1429-1430. doi:10.1038/sj.eye.6702289; published online 17 February 2006

Sir,

\section{Bilateral choroidal detachments due to massive} pulmonary embolism

Choroidal detachment (in the absence of haemorrhage) is caused by the transudation of serum into the suprachoroidal space. This is most commonly due to an increase in orbital transmural pressure, because of trauma, serum exudation, or inflammation. ${ }^{1}$ To the best of our knowledge, choroidal detachment is unreported in association with pulmonary embolism (PE). We report this previously serious occular complication of acute massive PE.

\section{Case report}

An 86-year-old man presented following a collapse outside his general practioner (GP) surgery. On arrival of the paramedics, he was cyanosed, with an unrecordable blood pressure. He was normotensive on arrival at hospital, where an initial electrocardiogram (ECG) showed acute right bundle branch block (RBBB). An arterial blood gas on high-flow oxygen showed hypoxia. The alveolar-arterial oxygen gradient was very high at 85.3.

A D-dimer was raised, as was the troponin.

A diagnosis of acute massive PE was made and he was anticoagulated with low molecular weight heparin and warfarin. He was not thrombolysed. A computed tomographic pulmonary angiogram (CTPA) scan, $12 \mathrm{~h}$ later, confirmed large bilateral pulmonary emboli.

He complained of blurred vision $24 \mathrm{~h}$ later. On examination, visual acuity was 6/60 in the right eye (RE) and light perception in the left intraoccular pressures (IOP) was normal. Pupils were reactive, but sluggish on the left. Anterior segment examination showed folds in the descements membrane. The anterior choroid was quiet. Dilated fundus examination revealed bilateral choroidal detachments with kissing choroids seen on B scan, with appearances consistent with suprachoroidal haemorrhage (Figure 1).

The patient was managed conservatively. After 10 days later, right visual acuity had improved to $6 / 18$, with counting fingers in the left eye (LE). By 1 month, there was near resolution of the right choroidal detachment, with an RE visual acuity of $6 / 6$ at 2 months. At 6 weeks following the initial insult, he developed a secondary LE vitreous haemorrhage, which failed to improve with conservative management. Anticoagulation was optimal throughout. He underwent a vitrectomy under local anaesthesia, leaving his LE visual acuity at 6/60 with resolved choroidal detachment, a flat retina, and no macular oedema.

\section{Comment}

Serous choroidal detachment involves the exudation of serum into the suprachoroidal space. There are several mechanisms for this, including low intraoccular pressure (IOP), which can be a result of ciliary body hypoperfusion. ${ }^{1}$ We consider the most likely mechanism for this patient's choroidal detachment to be a low arterial perfusion pressure. Acute massive PE leads to an acute rise in pulmonary artery pressure. This leads to reduced pulmonary blood flow and impaired left heart filling, leading to a low systemic blood pressure. ${ }^{2}$ The low cardiac output state leads to reduced carotid perfusion, and therefore reduced flow in the ophthalmic
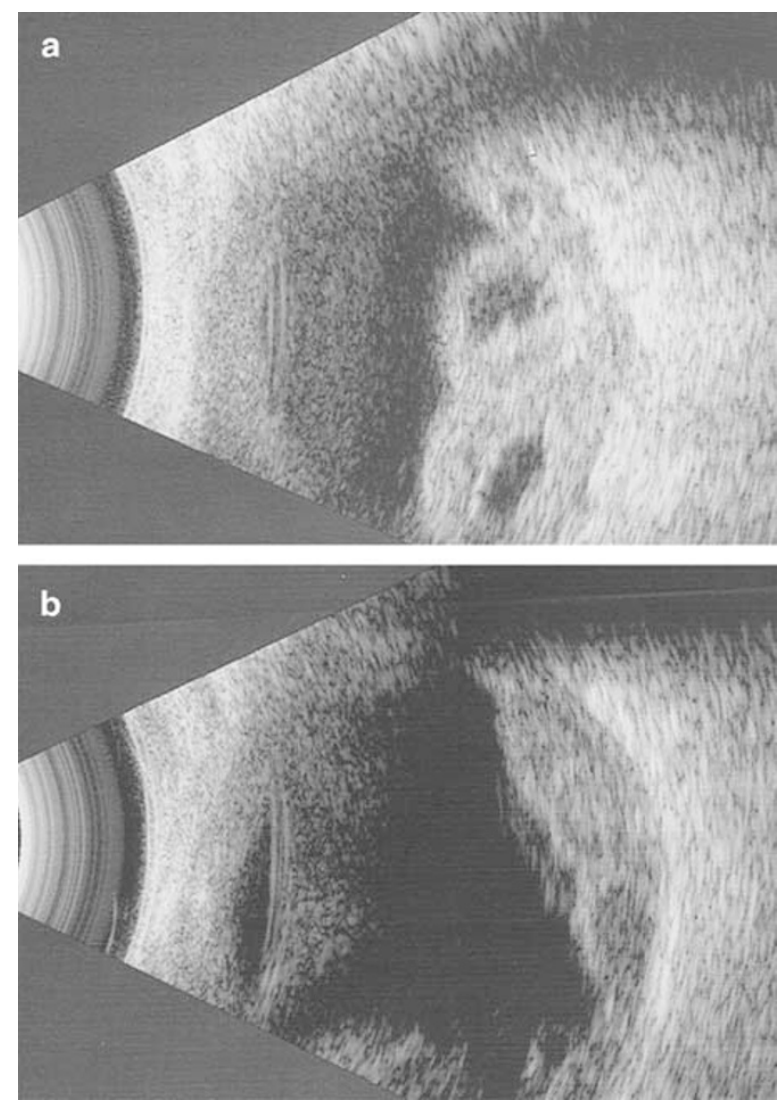

Figure 1 B scan of the RE (top panel, a) and LE (lower panel, b), 4 days post $\mathrm{PE}$, showing choroidal detachments with kissing choroids. 
artery, and other end arteries. ${ }^{1}$ The raised troponin on admission suggests secondary myocardial damage, ${ }^{3}$ again owing to a low perfusion pressure. His secondary LE vitreous haemorrhage is likely to be owing to a combination of warfarin anticoagulation in the presence of abnormal choroids. ${ }^{4,5}$

Complications of acute massive PE are rare in those who survive the initial insult. Small bowel hypoperfusion (presenting as fluid-filled dilated bowel loops - 'shock bowel') ${ }^{6}$ and cerebral hypoperfusion (presenting as fits) are reported owing to the systemic hypotension of acute PE. ${ }^{7}$ This is, to our knowledge, the first report of bilateral choroidal detachments in association with PE. We could not find any specific reason why this patient should suffer from this complication. Practitioners should be alert to signs of end organ damage following PE, and investigate promptly visual disturbance following a thromboembolic event.

\section{References}

1 Brubaker RF, Pederson JE. Ciliochoroidal detachment. Surv Ophthalmol 1983; 27: 281-289.

2 Feied CF. Pulmonary embolism. In: Rosen and Barkin (eds). Emergency Medicine Principles and Practice, 4th ed., $3^{\text {rd }} \mathrm{Vol}_{,}$ chapter 111, 1998.

3 Collinson PO, Chandler HA, Stubbs PJ, Moseley DS, Lewis $\mathrm{D}$, Simmons MD. Measurement of serum troponin T, creatine kinase $\mathrm{MB}$ isoenzyme, and total creatine kinase following arduous physical training. Ann Clin Biochem 1995; 32(Part 5): 450-453.

4 Narendran N, Williamson TH. The effects of aspirin and warfarin therapy on haemorrhage in vitreoretinal surgery. Acta Ophthalmol Scand 2003; 81: 38-40.

5 Edwards P. Massive choroidal hemorrhage in age-related macular degeneration: a complication of anticoagulant therapy. J Am Optom Assoc 1996; 67(4): 223-226.

6 De Backer AI, De Schepper AM, Hubens G, Vaneerdeweg W. Shock bowel following massive pulmonary embolism. J Belge Radiol 1997; 80: 231-232.

7 Marine JE, Goldhaber SZ. Pulmonary embolism presenting as seizures. Chest 1997; 113: 1732

A Gurbaxani1', GV Robinson², L Crawley', A Desai', J Wiggins $^{2}$ and J McAllister ${ }^{1}$

${ }^{1}$ Prince Charles Eye Clinic, King Edward VII

Hospital, Windsor, UK

${ }^{2}$ Department of General Medicine, Wexham Park

Hospital, Slough, UK

Correspondence: A Gurbaxani, King Edward VII Hospital, St Leonard Road, Windsor, Berks SL4 3DP, UK
Tel: + 440797729 6469;

Fax: + 4401753680441

E-mail: avigurbaxani@yahoo.co.in

Eye (2006) 20, 1430-1431. doi:10.1038/sj.eye.6702290; published online 3 March 2006

Sir,

Intraoperative floppy iris syndrome in a patient taking alfuzosin for benign prostatic hypertrophy

Intraoperative floppy iris syndrome (IFIS) is a recently described phenomenon affecting cataract surgery. It consists of poor preoperative pupil dilation together with progressive intraoperative pupil constriction, billowing of a flaccid iris stroma, and iris prolapse to the surgical incisions. IFIS has, to date, only been reported in patients symptomatic for benign prostatic hypertrophy $(\mathrm{BPH})$ treated with tamsulosin, a subtype a1A-selective adrenergic receptor blocker. ${ }^{1}$ We present a case of typical IFIS occurring in a patient taking alfuzosin, a nonsubtype-selective a1-adrenergic receptor blocker.

\section{Case report}

An 85-year-old man presented with age-related cataract. He had no other ocular findings of significance. He had been treated for 3 years with alfuzosin for symptoms of $\mathrm{BPH}$. He had never received any other a1-adrenergic receptor blockers. Small-incision phacoemulsification cataract surgery was planned. He was not asked to discontinue the alfuzosin before admission.

Preoperative pupil dilation was poor despite several instillations of cyclopentolate $1 \%$ and phenylephrine $2.5 \%$. Lidocaine $2 \%(2 \mathrm{ml})$ was injected into the subTenon's space inferonasally. A tunnelled temporal clear corneal incision was made. Iris behaviour typical of IFIS became apparent at hydrodissection. Disposable flexible translimbal iris retractors (Synergetics - Cat. No. Ref: 40.02) were therefore inserted in a diamond configuration similar to that described by Chang et al. ${ }^{1}$ Surgery was completed without further complications, with in-the-bag implantation of a flexible hydrophilic acrylic implant.

\section{Comment}

It has been postulated that a1A-subtype-selective adrenergic receptor antagonists cause IFIS by blocking 